\title{
STERILITY STUDIES. SIMPLIFIED METHODS IN DIAGNOSIS*
}

By William H. Cary, M.D., F.A.C.S., Brooklyn, N. Y.

Q IMPLICITY is art. If, therefore, by reviewing the facts adduced from several years of constant research in the subject of sterility we can finally arrive at a simplified method of studying the individual case which reduces the distasteful elemernls of such study to a minimum, both for the physician and for the patient, and also outline a short-cut procedure for determining the lesion interfering with conception, we shall feel that we have attained one of the essentials of a perfected technic.

The study of sterility has many discouraging features. Much clinical experience has been aceumulated and often it is contradictory. Exceptions are hard to account for; many preconceived impressions are reluctantly abandoned, and, finally, the percentage of cures which we can honestly claim, is small. Two years ago I was visited by a gynecologist of another city who asked me why I had published so little lately on the subject of sterility. I replied, with some emphasis, that I was discouraged with the work, that I knew little about it anyway, and did not propose to write until I had something definite to say.

While the general results of my work still seem meager, spectacular success occurs from time to time, and a review of the individual cases during recent years discloses facts about results which are interesting and promising.

My study showed that by far the larger proportion of patients were lopeless from the start. But hope springs eternal regarding fertility. Many patients were older than forty. Once a physician of sixty-five from a neighboring state, came with his wife of fifty-two. Another large group is made up of cases of male deficiency, and this finding frequently terminates the case. Not a small proportion of cases were those presenting frank histories of gonorrheal pathology. Of the latter group I am at present taking a more hopeful view, for reasons which I expect to outline in a later paper. Still a smaller group, but one I approach with little enthusiasm, is that of patients who give a suspicious history of morbidity following a useless curettage. Curetting has no place in the treatment of sterility except as a means of examination when symptoms suggest a diseased endometrium. Constantly I hear in my office, "The doctor found nothing wrong with me so he said he would operate and scrape the womb." The members of this society do not need to be instructed, but as teachers and consultants

*Read at a meeting of the New York Obstetrical Society, April 12, 1921. 
there is a moral responsibility upon us to warn the profession and the public of the dangers of the curet. My effort tonight will not be in vain if $\mathbb{T}$ have contributed something toward spreading this important precaution. The analysis of these groups shows definitely that a better. prognosis will be possible in sterility when the public and the profession are acutely aware of the need of early, competent study.

of necessity one's work in sterility will not, therefore, be brilliant, and $I$ advise and practice modesty in my prognosis to these anxious patients. Also I never tell a patient unreservedly that she cannot become pregnant (except when hysterectomy has been personally performed) for there is no mistake which may be so humiliating and disastrous.

The problem of sterility is usually first presented to the gynecologist and $I$ think it entirely proper and practicable that the initial study should begin with the gynecologic history and examination. This is not the place to discuss such elemental matters as history taking, but I do want to emphasize that I see no reasonable excuse for including in such a history any unusual questions of an intimate or embarrassing nature. Long ago I concluded that the knowledge gained by such inquiry was not pertinent to the subjeet and there is no surer way to discourage the earnest cooperation of the patient, which is such a valuable asset in the work. No other comment will be made upon the history. In the physical examination more than usual stress should be given to evidence of underdevelopment and endocrine disorders.

\section{THE EXAMTNATION}

Absolute classification of patients is impossible, but the gynecologic examination relative to sterility will disclose three general groups:

1. Cases with a gross pathology which entirely precludes pregnancy; or which renders pregnancy a dangerous complication. Also conditions which, while likely to cause sterility, have themselves an importance superseding sterility. This group of eases may be ruled out at once as not belonging to sterility problems.

2. The second group includes those patients with minor pelvic lesions, discovered incidentally, which have an importance due chiefly to the possible relation they bear to the interference of pregnancy. I refer to the flexions and uncomplicated versions of the uterus; socalled conical cervix with pinhole os; mucus plug obstructing the cervix; tilting of the cervix out of the seminal pool, et cetera. Much has been written as to their causative relation, but we are concerned with questions which actually determine their rôle in the individual patient. We may specifically state that the responsibility of such a lesion may be inferred only when the favorable character of all other essentials to fertility is demonstrated. When cases are thus studied, the contention of those who claim that these lesser pathologic condi- 
tions are very rarely responsible for sterility will be confirmed. They have been considered responsible, in my opinion, largely, because they are so often associated with sterility as the symptom-complex of underdevelopment.

3. In the third group may be placed those patients in whom the examiner fails to find any abnormality.

\section{THE INSEMINATION TEST}

The next stage of the study, applicable to the last two groups, is conducted on the second visit. I term it the "insemination test," and it consists simply of studying the condition and motility of the

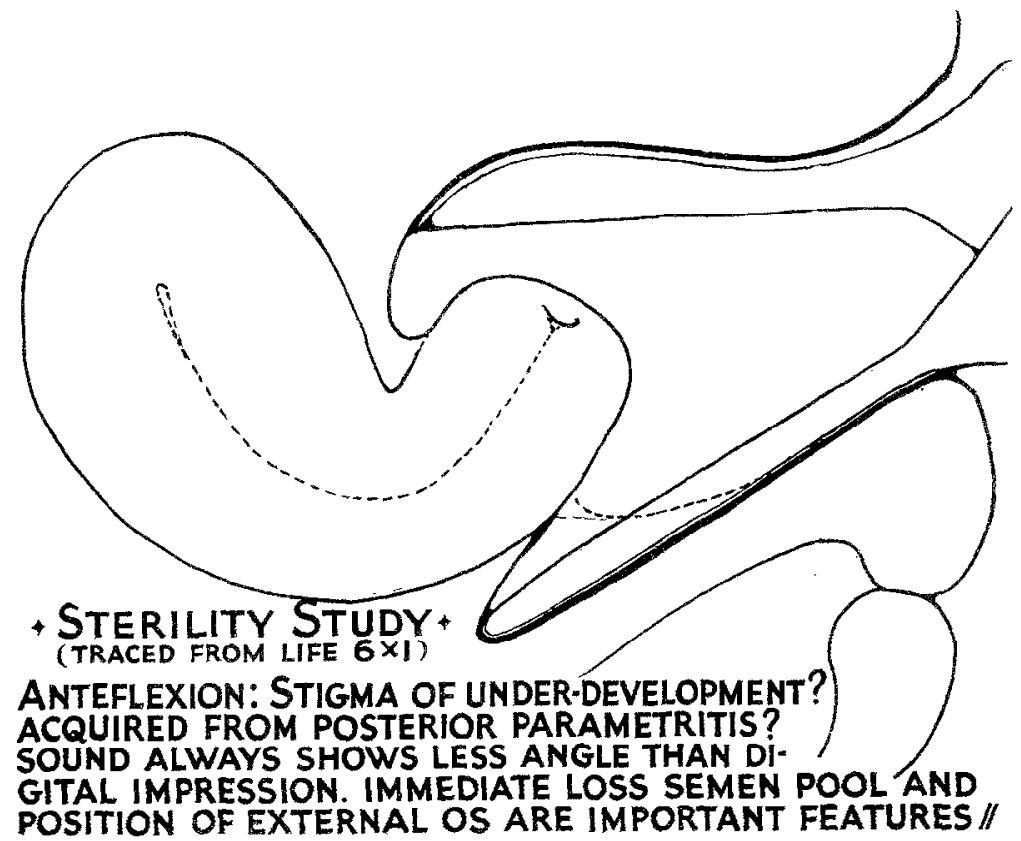

Fig. 1. "Figs, 1, 2 and 3 are after Ward's technic for cystopexy and amputation of the cervix.
Fig. 4 from Martin's Heftapparat.

spermatozoa in the reproductive tract of the recipient approximately forty minutes after coitus. Comparative findings are impossible without systematic study. This interval was originally specified as about the time necessary to permit the patient to reach the office. By test it was found also to present the most definitely favorable condition for study. I have found it practicable to set aside Sunday and one other morning a week for this work. The husband should be informed as to the nature of the investigation and a definite appointment for the wife's visit made a few days in advance to insure favorable cireumstances for study. Out-of-town patients avail themselves of a near-by hotel. A couple sincerely interested in childbearing will not offer ob- 
jection to a plan so easily executed and one devoid of artificial and distasteful features. I have gained acquiescence to this simplified program in every instance except one and the husband who objected related at my office the following day a history of double epididymitis just before marriage. At the appointed hour I have ready on a table, beside the examining table, a warm microscope and a warmed tray, which I call the sterility tray, which contains the following articles: clean glass slides with blank labels, cover glasses, litmus paper, and numerous pipettes of various types and sizes including Skene's pipettes, some of which I have modified so that they may be most useful for obtaining specimens from the cervical canal, or for injection in the tube patency test which I shall describe later.

The patient, having arrived at the appointed hour, is prepared, with loosened corset, in the usual dorsal position. The vagina and cervix are exposed with a warm speculum introduced without lubricant, for obvious reasons. The examination should proceed at once. Under normal conditions, the examiner will find a pool in the vaginal sulcus varying greatly in amount, but averaging about a dram, consisting of seminal elements mixed with vaginal secretion. The pool is thin in density; slightly alkaline in reaction; and when transferred to the microscope shows a field swarming with vigorously moving spermatozoa amid vaginal and seminal debris. To avoid errors of contamination, the vagina is now dried and if mucus is protruding from the cervical os a specimen is taken for examination. This mucus is then removed and specimens of mucus taken from the cervical canal up to the internal os. Some difficulty may be experienced in obtaining tenacious mucus, but with patience and a trial of different pipettes, avoiding roughness that causes oozing, specimens will be obtained which, when spread under a cover glass, will show from four to sixteen active sperm cells to a field. Cells which have made this progress to the cervical canal are almost invariably normal as to morphology and mobility showing Nature's simple plan that only the fittest shall survive to perpetuate the species. Vaginal elements are sometimes found in the cervical canal. In connection with this, I wish to say that a peristaltic action of the uterus as a phenomenon of the orgasm seems proved, for, in experimental study I have found spermatozoa with vaginal epithelium, and other elements well within the cervical canal immediately after intercourse. Thus may be explained the contradictory experience as to the value of the douche as a contraceptive measure.

\section{DEFECTIVE INSEMINATION}

The absence of the male element in the vagina or the presence of seminal fluid but with few or no spermatozoa, establishes at once the male responsibility and the problem is transferred to the genito- 
urinary surgeon. Again, if the examination demonstrates a normal pool retention with the usual sperm content but without motility or with few active cells; or with sluggish activity with decreased or absent motility in the cervical canal only two conclusions are possible; either the male element has been deficient from the first or, having been normal, has been adversely affected by the vaginal secretion. To determine which is the correct hypothesis our investigation must be temporarily diverted to the husband. A specimen obtained directly in one of the methods described previously before this society, ${ }^{1}$ is examined after the lapse of forty minutes just mentioned. Gross errors will result if this technic is careless; but if correctly carried out, one may reach conclusions with confidence. It was some time before I acquired the courage to ascribe sterility to male specimens of partial deficiency. I have nothing of greater importance to state tonight than that whilc a specimen with some motility is theoretically capable of impregnation, experience has shown that pregnancy does not occur if the male product is definitely weak. Many couples come to me with the statement that the husband has been examined and is "all right." In checking up this finding I am convinced that the average examination proceeds no further than the demonstration of some active cells. One husband said he had made a diagnosis himself of normality, for, after the doctor put the specimen under the microscope, he plainly saw things wiggling. Cooperation of an associate in genitourinary diseases will invariably show vital anatomic or functional troubles in the men hav. ing decreased activity of spermatozoa and there is no reason to modify the statement, made some years ago, that when our investigation has proceeded thus far-practically after two visits-fully fifty to sixty per cent of the cases will be eliminated as gynecologic problems.

If the male specimen obtained directly proves fertile and a second insemination test shows its vitality to be again destroyed, the adverse action is shown to be definitely in the vagina. In our experience this has always been due to hyperacidity though others have ascribed it to a toxicity of bacterial origin. The latter is a reasonable assumption and worth investigation. Hyperacidity may be assumed, if the vaginal pool is found definitely acid. Spermatozoa soon die in such a medium. Hyperacidity is a relative term in sterility and we do not ascribe to it the importance which was formerly noted. This test, however, af. fords a means of fixing its responsibility-definite and destructive in occasional instances. The spectacular cures sometimes following alkaline douches are thus accounted for, but the reverse does not hold good that hyperacidity is ruled out by the haphazard use of bicarbonate of soda or sodium hydrate douches, for marked vaginal acidity is not so easily corrected.

When in cases of the second group a normal semen is demonstrated 
and the patient is free from any suspicion of pelvic inflammatory changes, one is justified in studying and correcting these minor lesions as a possible etiologic factor. No discussion regarding treatment shall be given here. Flexions and other anatomic signs should encourage investigation of delayed development and appropriate treatment. There are more cases of this type than is commonly thought. Correcting a version may clear up an endometritis or some other unfarorable condition. Cures will certainly follow in a certain proportion of cases from the use of a pessary; or from dilatation of the cervix, with or without stem insertion, and for reasons that are not always definitely explainable.

When the minor lesion of a patient in group two is corrected for several months without pregnancy oceurring, the patient automatically belongs in group three, that is, patients without demonstrable pathology. When spermatozoa are traced in normal activity to the internal os we may assume that in these patients sterility is due either to deficient ovum production, or a blockade in the fallopian tubes, or, possibly, a change in the endometrium which prohibits growth. Very frequently indeed, upon the conclusion of the test at the second visit of the patients, who formerly were particularly puzzling because so normal, the etiology of the sterility may be fixed within these narrow limits. Delay in development; illness or hard wor]x during the years of puberty; absence of normal instincts; disturbed function, such as scant menses or intermittent amenorrhea may strongly point to endocrine deficiency, involving the ovary, but not conclusively proving absence of ovulation. The study of this relation offers an opportunity for valuable research.

In by far the largest percentage of cases in this group the lesion is in the fallopian tubes. It seems we have been grievously slow when studying these cases to recognize that the tubes are frequently sealed by gradual processes, other than gonorrheal or tuberculous, and without marked symptoms or palpable pathology. Probably each of you in performing a laparotomy has often had the experience of finding sealed tubes in patients who gave not the slightest suspicion of it either in the history or the vaginal examination. Many of these obstructions probably develop from the action of the colon bacillus.

Appreciating the difficulty of recognizing this important lesion, I proposed some years ago to prove the patency of the tubes by radiography after collargol injections into the tubes. ${ }^{2}$ This proved impractical, but suggestive. If we cannot claim that this problem is already solved, at least the study is well on the way. Rubin has proposed, and is using, a most ingenious method of proving tubal patency by demonstrating the injection of oxygen into the peritoneal cavity 
through the uterus. I have for some time been developing another method, similar in principle, by demonstrating the injection of sterile normal saline solution through the tubes by carefully measuring the amount injected in excess of the 8 or 10 minims known to be the capacity of the uterine cavity. In other words, by experimental injection through the uterus, just before laparotomy, we found that the reception of 16 minims of solution means escape through a tube. The possibility of such a test was first suggested to me in attempting artificial impregnation. In some patients the injection proceeded easily with definite peritoneal reaction. In others there was an equally definite limitation to the injection and almost no progress was made. After a little experience, the ease with which the solution is injected is as significant as the amount introduced.

When by a process of exclusion, as represented by the insemination test, the cause of sterility is practically limited to the tube or ovary, the history of associated findings will usually make the differential diagnosis possible. In my opinion the necessity of proving the patency of the tubes will be exceptional. I limit the test to the most carefully selected patients who give no definite past history of pelvic inflammatory disease and when two pelvic examinations have caused no reaction.

\section{TECHNIC OF IN.JECTION TEST}

The injection test has simplicity to commend it. Freshly made normal saline solution is used and is boiled immediately before injection. Pipettes with the Skene curved tip of varying sizes, are used. The pipette is ehosen which snugly fits the individual patient and is carefully sterilized. Sixteen minims of the solution are taken up in the pipette. The patient with emptied bladder and removed corset is placed in a perfect knee chest position and the cervix exposed with the Sims speculum. The vagina is wiped dry and the external os sponged free of mucus. Mucopurulent material in the canal is considered a contraindication to the test. Strips of dry paper are placed in the most dependent part of the vagina to aid the eye in detecting escape of the solution around the pipette. The pipette is now snugly engaged in the canal of the ungrasped cervix. Gentle pressure is then exerted upon the rubber bulb, tightly tied to avoid leak, and a headlight fixed upon the pipette and cervix. Moderate pressure is steadily exerted and the patient instructed to take a deep breath. It is surprising how readily the saline disappears if either tube be open. If they are closed no headway will be made; the pipette will be removed with most of the saline still in it, or leakage will occur about the pipette which, even if undetected by the eye, will soak the tell-tale strips of paper. No immediate 
discomfort to the patient follows the successful injection, but about three hours later a definite peritoneal reaction occurs with some pain in the nature of a colic. Patients are told to expect this and to stay in bed over the anticipated period. Larger amounts of saline are unnecessary and are cautioned against because of the more painful reaction.

That this test, applied for purposes of diagnosis, may possibly also be responsible for the opening of a sealed tube, was demonstrated in the patients, previously referred to, who were laparotomized after an injection into the tubes. I am of the opinion that, except in vaginal or cervical disease, when artificial impregnation succeeds it does so because occasionally a blocked tube is thus opened. This is the only

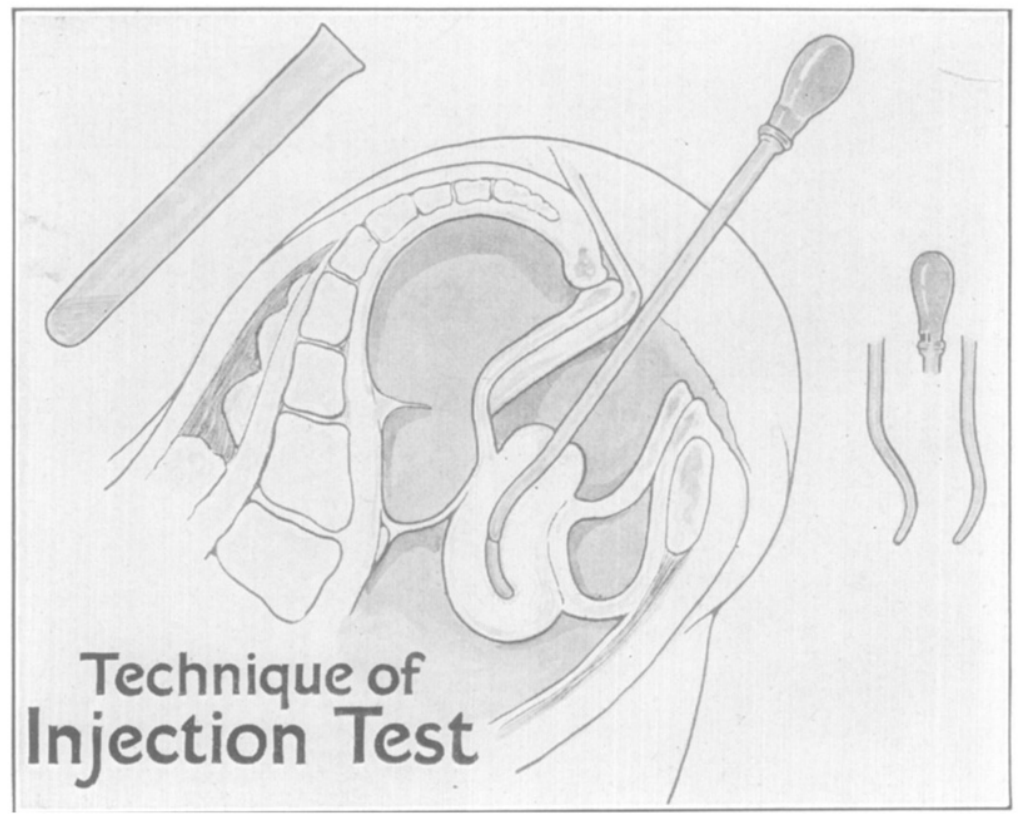

Fig. 2.

rational explanation and for this reason I have practically abandoned attempts at artificial impregnation except to facilitate the passage of spermatozoa through the cervical canal. If my opinion is correct, normal saline is certainly safer for purposes of injection than is seminal fluid: The possibility of carrying infection to the peritoneal cavity by this method is recognized if the technic be careless and the cases improperly chosen. If, however, we have confidence in the peritoneum allowing us to close the abdomen without drainage after hysterectomy, incising across the cervix or vaginal cuff, and if we contaminate the peritoneum with the contents of tubes with impunity, why should our confidence in the peritoneum be abandoned in the instance of this lesser danger ? 
414 THE AMERICAN JOURNAL OF OBSTETRICS AND GYNECOLOGY

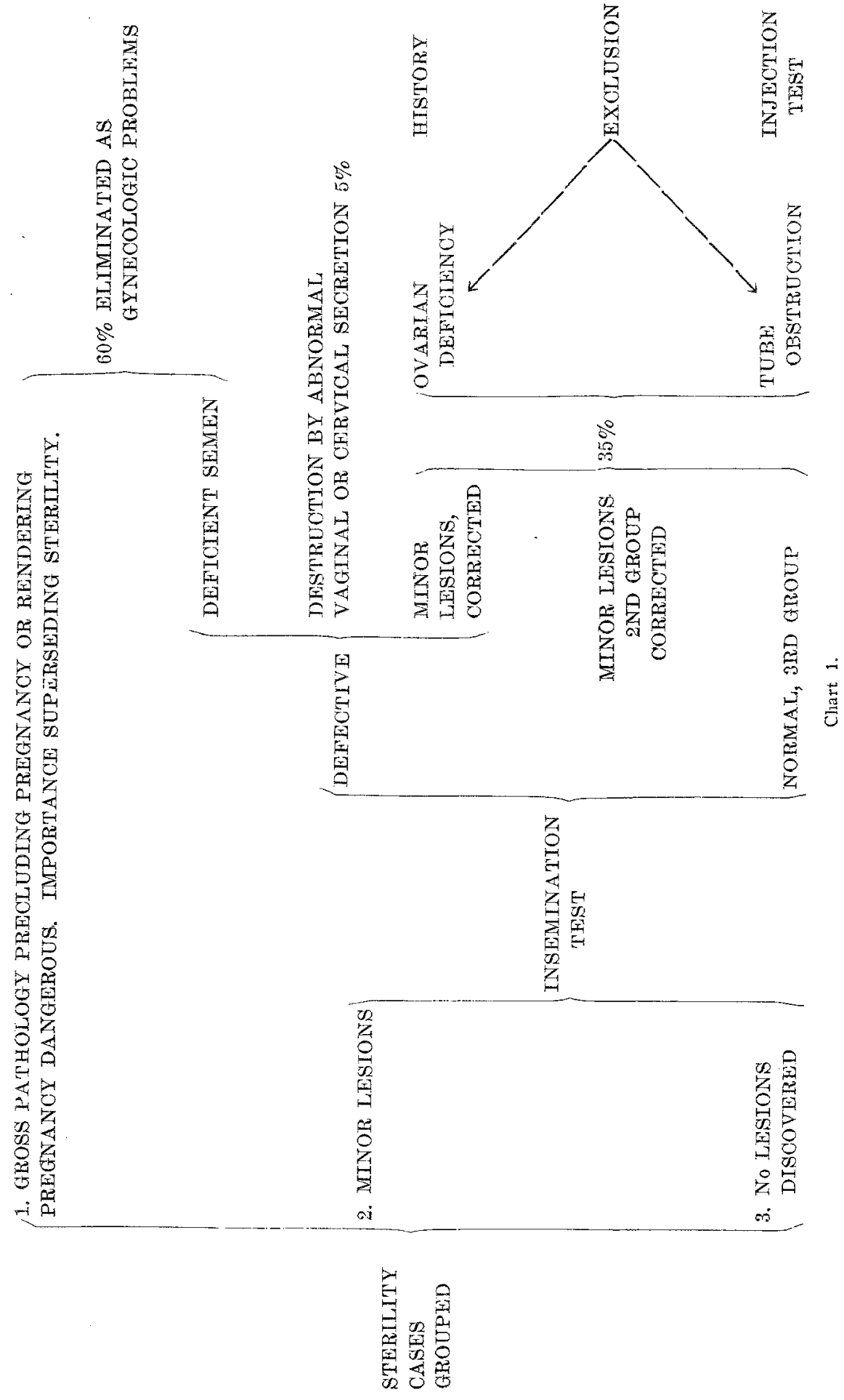


CONCLUSIONS

While one's results in treating sterility may not be brilliant, the prognosis would be greatly improved if patients sought competent study earlier.

The indiscriminate use of the curette in the treatment of sterility is to be emphatically and unconditionally condemned. In the hands of an expert it may be condoned as an instrument of diagnosis ; rarely for treatment.

Further study confirms that the husband shares equally the responsibility for sterility. Casual reports of male virility are not to be accepted. Sterility may be attributed to partial defects of the semen. These cases offer an excellent prognosis.

A simplified method of studying sterility, original ${ }^{3}$ only in its application, is presented as outlined in the accompanying chart. It eliminates most of the unpleasant features of previous methods and enables a quick and definite diagnosis of the inhibiting lesion.

Finally, a technic, for proving the patency of the fallopian tubes in carefully selected cases, is elaborated, which is so simple as to be available to any gynecologist of ordinary training and equipment.

REFERENCES

(1) Am. Jour. Obst., 1916, lxxiv, No. 4 (2) Am. Jour. Obst., 1914, lxix, No. 3. (3) Hiinner, M.: Practical Treatise on Disorders of Sexual Functions in the Male and Female, 1916.

(For discussion, see page 440.) 